\title{
COMMENTARY
}

\section{Finding new therapies for sepsis: the need for patient stratification and the use of genetic biomarkers}

\author{
Michael J Hobson and Hector R Wong* \\ See related research by Turrel-Davin et al., http://ccforum.com/content/15/5/R252
}

\begin{abstract}
Reversing the immunoparalysis associated with septic shock remains a priority for improving the outcome of patients suffering from sepsis. The efficacy of future therapies may be better studied under an effective system of patient stratification. Gene expression biomarkers offer a mechanism by which patients may be appropriately stratified in such clinical trials.
\end{abstract}

Sepsis remains a leading cause of morbidity and mortality in critically ill patients. In a subgroup of patients, morbidity and mortality are linked to immunoparalysis that ensues after the initial inflammatory cascade of septic shock [1,2]. Accordingly, therapies to restore proper immune function hold promise in the treatment of immunoparalysis-related morbidity. However, successful implementation of these therapies depends on the recognition of sepsis as a heterogeneous clinical syndrome and the ability to identify (stratification) patients with immunoparalysis. Several factors account for sepsis heterogeneity: the infectious organism and focus of infection, host genetics and its effect on the inflammatory response, and co-morbidities [3]. Conversely, experimental treatments in sepsis clinical trials have historically been homogeneously applied without adequately addressing this heterogeneity, and this likely has contributed to the lack of efficacy seen in most clinical trials [4].

Moving forward, successful application of immunomodulating therapies hinges upon identifying and accounting for the biological variation among patients

\footnotetext{
*Correspondence: hector.wong@cchmc.org

Division of Critical Care Medicine, Cincinnati Children's Hospital Medical Center and Cincinnati Children's Research Foundation, Department of Pediatrics, University of Cincinnati College of Medicine, Cincinnati, OH 45229-3039, USA
}

with sepsis. Such a stratification or staging system would serve to mitigate the issues surrounding disease heterogeneity, while simultaneously allowing a potential therapy to target a more distinct, biologically appropriate population. Under this design, the potential efficacy of a new therapeutic strategy is not attenuated by its use in patients who are not primed or predisposed to receive benefit [5].

An important challenge in the field, then, lies in selecting the parameters or biological 'readouts' that will be used for stratification, as has been attempted by Turrel-Davin and colleagues in the previous issue of Critical Care [1]. Illness severity scores are not appropriate, nor are intended, for individual patient stratification [6]. Biomarkers may serve both as a means for selecting patients eligible for immunomodulatory therapies, as well as for monitoring response to treatment. Stratification biomarkers can identify high risk patients potentially eligible for higher risk therapies [4]. Likewise, these same biomarkers could be used to exclude patients who are projected to have a good clinical outcome with standard therapy. The goal of stratification would thus be to target a distinct biological process, while at the same time minimizing the risk-benefit ratio.

Gene expression profiling has been used to discover sepsis-related biomarkers. It is unlikely that a single drug or intervention will ever come as the end-all treatment for septic shock, and thus broad-scale tools may allow us to stratify a patient's immune status and consequently allow for more objective selection of immune modulating therapies [7]. Recently we have shown that gene signatures and gene expression mosaics obtained from patients within the first 24 hours of septic shock can be used to stratify patients into subclasses having their own particular severity of illness, rate of organ failure, and mortality [8]. This work shows that gene expression methods may represent a robust and achievable process by which patients with sepsis can be stratified. Similarly, Turrel-Davin and colleagues have shown that mRNA 
expression can be used to monitor subsequent response to treatment [1].

While biomarkers appear to hold promise for staging strategies in the future, such optimism must be met with caution. The biologic model under which biomarkers are derived may have a significant impact on the conclusions that we draw about a particular biomarker. For example, in the study by Turrel-Davin and colleagues, the presence of low-dose lipopolysaccharide in cell culture induced endotoxin tolerance, and this subsequently resulted in an attenuated inflammatory response following a second lipopolysaccharide challenge at a higher dose. In contrast, in a murine model where lipopolysaccharide-induced endotoxin tolerance was followed by cecal ligation and puncture (that is, an in vivo infectious challenge), endotoxin tolerance enhanced bacterial clearance and improved animal survival [9]. Thus, it is not entirely clear whether the phenomenon of endotoxin tolerance is biologically equivalent to the immunosuppression observed in critically ill patients. Second, the demographics of the patient population being studied may influence biomarker derivation and performance. For example, plasma interleukein-8 levels have been shown to robustly predict survival in pediatric patients with septic shock [10]; however, this same biomarker is not an effective risk stratification tool when applied to older adults [11]. Likewise, Turrel-Davin and colleagues showed gene expression restoration after recombinant interferon gamma treatment of monocytes obtained from elderly (all over the age of 64 years) patients with septic shock [1]. We must be cautious in extrapolating these findings to younger patient populations.

Advancements in the treatment of septic shock may lie in the ability to tailor therapies according to the specific immune status of an individual patient (stratification). The discovery and implementation of these therapies are most likely to be successful if studied in more biologically homogeneous patient populations. The use of gene expression biomarkers may allow us to identify these patients as well as to follow their response to therapy.
Such rationale should hold promise in the future development of tools used for treating our patients suffering from sepsis.

\section{Competing interests}

HRW and Cincinnati Children's Hospital Medical Center have submitted a provisional patent application for the use of interleukin-8 as a stratification tool in pediatric septic shock. MJH declares that he has no competing interests.

Published: 7 December 2011

\section{References}

1. Turrel-Davin F, Venet F, Monnin C, Barbalat V, Cerrato E, Pachot A, Lepape A, Alberti-Segui C, Monneret G: mRNA-based approach to monitor recombinant gamma-interferon restoration of LPS-induced endotoxin tolerance. Crit Care 2011, 15:R252.

2. Hotchkiss RS, Opal S: Immunotherapy for sepsis - a new approach against an ancient foe. N Engl J Med 2010, 363:87-89.

3. Marshall JC, Reinhart K: Biomarkers of sepsis. Crit Care Med 2009, 37:2290-2298.

4. Kaplan JK, Wong HR: Biomarker discovery and development in pediatric critical care medicine. Pediatr Crit Care Med 2011, 12:165-170.

5. Marshall JC: The staging of sepsis: understanding heterogeneity in treatment efficacy. Crit Care 2005, 9:626-628.

6. Vincent JL, Opal SM, Marhsall JC: Ten reasons why we should not use severity scores as entry criteria for clinical trials or in our treatment decisions. Crit Care Med 2010, 38:283-287.

7. Feezor RJ, Cheng A, Paddock HN, Baker HV, Moldawer LL: Functional genomics and gene expression profiling in sepsis: beyond class prediction. Clin Infect Dis 2005, 41(Suppl 7):S427-S435.

8. Wong HR, Wheeler DS, Tegtmeyer K, Poynter SE, Kaplan JM, Chima RS, Stalets E, Basu RK, Doughty LA: Toward a clinically feasible gene expression-based subclassification strategy for septic shock: proof of concept. Crit Care Med 2010, 38:1955-1961.

9. Wheeler DS, Lahni PM, Denenberg AG, Poynter SE, Wong HR, Cook JA, Zingarelli B: Induction of endotoxin tolerance enhances bacterial clearance and survival in murine polymicrobial sepsis. Shock 2008, 30:267-273.

10. Wong HR, Cvijanovich N, Wheeler DS, Bigham MT, Monaco M, Odoms K, Macias WL, Williams MD: Interleukin-8 as a stratification tool for interventional trials involving pediatric septic shock. Am J Respir Crit Care Med 2008, 178:276-282.

11. Calfee CS, Thompson BT, Parsons PE, Ware LB, Matthay MA, Wong HR: Plasma interleukin-8 is not an effective risk stratification tool for adults with vasopressor-dependent septic shock. Crit Care Med 2010, 38:1436-1441.

do: $10.1186 /$ cc10527

Cite this article as: Hobson MJ, Wong HR: Finding new therapies for sepsis: the need for patient stratification and the use of genetic biomarkers. Critical Care 2011, 15:1009. 